\title{
Adaptive Discrete-time Fuzzy Sliding Mode Control For a Class of Chaotic Systems
}

\begin{tabular}{|c|c|}
\hline \multicolumn{2}{|c|}{ Hanene Medhaffar $\bigsqcup^{\text {}} 1$, Moez Feki ${ }^{2}$, Nabil Derbel ${ }^{1}$} \\
\hline \multicolumn{2}{|c|}{${ }^{1}$ Research Unit ICOS, CEMLab, Sfax Engineering School, University of Sfax, Tunisia } \\
\hline \multicolumn{2}{|c|}{$\begin{array}{l}{ }^{2} \text { Laboratoire de Mathématiques: Modélisation Déterministe et Aléatoire (LAMMDA), } \\
\text { ESSTHS, University of Sousse, Tunisia }\end{array}$} \\
\hline A R T I C L E I N F O & A B S T R A C T \\
\hline Article history: & In this paper, we propose an adaptive discrete-time fuzzy sliding mode \\
\hline Received: 04 April, 2017 & control for a class of chaotic systems. For this aim, a discrete sliding \\
\hline Accepted: 01 May, 2017 & mode controller and a fuzzy system are combined to achieve an \\
\hline Online: 22 May, 2017 & adequate control. The Laypunov stability theorem is used to testify the \\
\hline Keywords: & asymptotic stability of the whole system and the consequence \\
\hline Sliding mode control & parameters of the adaptive fuzzy system are tuned on-line by adaptive \\
\hline Adaptive fuzzy system & laws. The simulation example of the $3 D$ Henon chaotic model is giving \\
\hline Discrete time chaotic system & to confirm the effectiveness and the robustness of the proposed method. \\
\hline
\end{tabular}

\section{Introduction}

In recent years, the control of chaotic systems has increasingly interested researchers. The first chaos control method has been proposed by Ott et al. [1], nowadays known as the OGY (Ott-Grebogi-Yorke) method. This is a discrete technique that considers small perturbations applied in one system parameter when the trajectory approaches the vicinity of the desired orbit when crossing a specific surface. Since then, numerous control techniques have been proposed for controlling chaos in different chaotic systems such as backstepping [2-4], adaptive control algorithms [5-7] and sliding-mode control [8-10].

The sliding mode control (SMC) has undergone extensive and detailed studies in the last three decades. It is noted that SMC is a powerful robust control strategy treating the model uncertainties and external disturbances. The design and the implementation of discrete time sliding mode control have later been considered, and still in progress because a large classes of continuous systems are controlled by digital signal processors and microprocessors. Indeed, discrete sliding mode control is well studied in the literature [11-14]. The aim of this work is to give a further contribution in this field. The main objective is to design a discrete time sliding mode control strategy and enhanced by a stable adaptive fuzzy inference system to cope with modeling inaccuracies and external disturbances that can arise. Many researches on introducing the concept of fuzzy logic and especially fuzzy approximators into sliding mode control have been de- veloped in the past years [15-18]. Historically, fuzzy logic systems have been proposed in an attempt to control nonlinear systems whose parameters are inaccuracy, and presenting neglected dynamics as well as time varying systems [19]. Since then, fuzzy logic control becomes an active research area and it has been implemented in several industrial applications. Accordingly, fuzzy logic with discrete sliding mode control are proposed to improve system control performances. Thus, the overall control system drives the tracking error to zero even in the presence of external disturbances. This paper is organized as follows: Section 2 and 3 deal with discrete sliding mode and fuzzy system respectively. Moreover, the detailed design procedure of fuzzy sliding mode controller is explained in section 4 . Numerical simulations are carried out in section 5 for illustration and verification of the proposed controller. Finally some concluding remarks are given in section 6 .

\section{Discrete-time sliding mode con- troller}

Consider the following nonlinear discrete time system

$$
\left\{\begin{array}{c}
x_{1}(k+1)=x_{2}(k) \\
x_{2}(k+1)=x_{3}(k) \\
\vdots \\
x_{n-1}(k+1)=x_{n}(k) \\
x_{n}(k+1)=f(x(k))+u(k)+d(k)
\end{array}\right.
$$


where $x(k)=\left[x_{1}(k), x_{2}(k), \ldots, x_{n}(k)\right]^{T}$ is the state vector and $u(k)$ is the input signal.

$f(x(k))$ is unknown but it is a bounded function and $d(k)$ is a bounded external disturbance such that $|d(k)|<D$.

When $u(k)=0$, system (1) behaves chaotically. Therefore, the aim of this work is to apply a discrete-time sliding mode controller $u(k)$ in order to track a desired trajectory.

Let $x_{d}(k)=\left[x_{d 1}(k), x_{d 2}(k), \ldots, x_{d n}(k)\right]^{T}$ the well known desired trajectory. Then, the tracking error can be expressed as

$$
e(k)=x(k)-x_{d}(k)
$$

where $e(k)=\left[e_{1}(k), e_{2}(k), \ldots, e_{n}(k)\right]^{T}$.

The sliding surface can be defined as

$$
\begin{aligned}
s(k) & =c_{1} e_{1}(k)+c_{2} e_{2}(k)+\ldots+c_{n-1} e_{n-1}(k)+e_{n}(k) \\
& =C e(k)=0
\end{aligned}
$$

where $C=\left[c_{1}, c_{2}, \ldots, c_{n-1}, 1\right]$ can be selected as $h(z)=$ $z^{n-1}+c_{n-1} z^{n-2}+\ldots+c_{2} z+c_{1}$ is stable. The sliding mode controller is designed by adopting the reaching law defined by [12]

$$
\Delta s(k+1)=s(k+1)-s(k)=-Q s(k)-K \operatorname{sign}(s(k))
$$

where $0<Q<1$ and $K>0$.

If $f(x(k))$ is supposed known and $d(k)=0$ then

$s(k+1)=\sum_{i=1}^{n-1} c_{i} e_{i}(k+1)+f(x(k))+u(k)-x_{d n}(k+1)$

therefore $\Delta s(k+1)$ can be expressed as

$$
\begin{aligned}
\Delta s(k+1) & =\sum_{i=1}^{n-1} c_{i} e_{i}(k+1)+f(x(k))+u(k) \\
& -x_{d n}(k+1)-\sum_{i=1}^{n-1} c_{i} e_{i}(k)-e_{n}(k)
\end{aligned}
$$

(6) where

The equivalent control $u_{e q}(k)$ can be derived by using $\Delta s(k+1)=0$ such that

$$
\begin{aligned}
u_{e q}(k) & =-\sum_{i=1}^{n-1} c_{i} e_{i}(k+1)-f(x(k))+x_{d n}(k+1) \\
& +\sum_{i=1}^{n-1} c_{i} e_{i}(k)+e_{n}(k)
\end{aligned}
$$

Since $d(k) \neq 0$ then a switching type control must be added such that

$$
\begin{aligned}
u(k) & =u_{e q}(k)+u_{s}(k) \\
& =-\sum_{i=1}^{n-1} c_{i} e_{i}(k+1)-f(x(k))+x_{d n}(k+1) \\
& +\sum_{i=1}^{n-1} c_{i} e_{i}(k)+e_{n}(k)+u_{s}(k)
\end{aligned}
$$

$$
\theta=\left[\begin{array}{c}
y^{1}(x(k)) \\
: \\
y^{H}(x(k))
\end{array}\right], \xi(x(k))=\left[\begin{array}{c}
\xi^{1}(x(k)) \\
: \\
\xi^{H}(x(k))
\end{array}\right]
$$

where $u_{s}(k)$ is defined by

$$
u_{s}(k)=-Q s(k)-K \operatorname{sign}(s(k))
$$

where the switching gain $K$ will be determined afterwards .

Furthermore, if $f(x(k))$ is unknown then a fuzzy system $\hat{f}(x(k))$ will be used to approximate $f(x(k))$ in order to obtain the sliding mode control law. Moreover, an adaptive adjusting law will be designed.

\section{Fuzzy system}

knowledge base for the fuzzy logic system com-

$$
\begin{aligned}
& \mathbf{R}_{\mathbf{1}}^{(\mathbf{j})}: \text { If }\left[\left(x_{1}(k) \text { is } A_{1}^{j}\right) \ldots \text { and }\left(x_{n}(k) \text { is } A_{n}^{j}\right)\right] \\
& \text { then }\left[\left(y(x(k))=b^{j}\right)\right]
\end{aligned}
$$

pour $j=1, \ldots, H . \mathrm{H}$ is the rule number of the fuzzy logic system.

$x_{i}(k), i=1, \ldots, n$ and $y(x(k))$ denote the linguistic variables associated with the inputs and the output of the fuzzy logic system.

By the use of the singleton fuzzification strategy, product inference and center-average defuzzification, the output of the fuzzy system is expressed as:

$$
y(x(k))=\frac{\sum_{j=1}^{H} y^{j}(x(k))\left(\prod_{i=1}^{n} \mu_{A_{i}^{j}}\left(x_{i}(k)\right)\right)}{\sum_{r=1}^{H}\left(\prod_{p=1}^{n} \mu_{A_{p}^{r}}\left(x_{p}(k)\right)\right)}
$$

where $\mu_{A_{i}^{j}}$ is the membership function of the linguistic variable $A_{i}^{j}$.

By introducing the concept of fuzzy basis function vector $\xi(x(k)), 11$ can be rewritten as:

$$
y(x(k))=\theta^{T} \xi(x(k))
$$

and

$$
\xi^{j}(\mathbf{x})=\frac{\prod_{i=1}^{n} \mu_{A_{i}^{j}}\left(x_{i}(k)\right)}{\sum_{r=1}^{H}\left(\prod_{p=1}^{n} \mu_{A_{p}^{r}}\left(x_{p}(k)\right)\right)}
$$

$\xi^{j}(x(k))$ which are called fuzzy basis functions (FBF's). It has been proved that these FBF's are universal approximators [20].

\section{Fuzzy Sliding mode control}

In order to derive the sliding mode control, the fuzzy system $\hat{f}\left(x(k) / \theta_{f}\right)$ is used to approximate $f(x(k))$ [21]. The fuzzy logic system $\hat{f}\left(x(k) / \theta_{f}\right)$ is expressed by:

$$
\hat{f}\left(x(k) / \theta_{f}\right)=\theta_{f}^{T} \xi_{f}(x(k))
$$


where $\xi_{f}(x(k))$ is the vector of fuzzy basis supposed to be fixed, while parameters $\theta_{f}$ are variables which will be designed by adaptive laws.

Let $\theta_{f}^{*}$ the optimal parameter vectors of the fuzzy logic system. Minimum approximation error can be defined as follows:

$$
w_{f}(k)=f(x(k))-\hat{f}\left(x(k) / \theta_{f}^{*}\right)+d(k)
$$

Then, we can select the control law as:

$$
\begin{aligned}
u(k) & =u_{e q}(k)+u_{s}(k) \\
& =-\sum_{i=1}^{n-1} c_{i} e_{i}(k+1)-\hat{f}\left(x(k) / \theta_{f}\right)+x_{d n}(k+1) \\
& +\sum_{i=1}^{n-1} c_{i} e_{i}(k)+e_{n}(k)-Q s(k)-K \operatorname{sign}(s(k))(1
\end{aligned}
$$

where $0<Q<1$ and $K$ will be determined afterwards. Therefore, $\Delta s(k+1)$ can be rewritten as

$$
\begin{aligned}
\Delta s(k+1) & =f(x(k))-\hat{f}\left(x(k) / \theta_{f}\right)-Q s(k)-K \operatorname{sign}(s(k)) \\
& =\hat{f}\left(x(k) / \theta_{f}^{*}\right)-\hat{f}\left(x(k) / \theta_{f}\right)+w_{f}(k) \\
& -Q s(k)-K \operatorname{sign}(s(k)) \\
& =\Phi_{f}^{T}(k) \xi_{f}(x(k))+w_{f}(k) \\
& -Q s(k)-K \operatorname{sign}(s(k))
\end{aligned}
$$

where $\Phi_{f}(k)$ represent the fuzzy parameter errors such that:

$$
\Phi_{f}(k)=\theta_{f}^{*}-\theta_{f}(k)
$$

Theorem 1 The following adaptive law for adjusting the parameter vector $\theta_{f}$

$$
\Delta \theta_{f}(k)=-\alpha \xi_{f}(x(k)) s(k)
$$

asymptotically stabilizes system (1) controlled by (17), where $\alpha$ is a positive constant which determines the rate of adaptation.

Proof: The Lyapunov function candidate is chosen as

$$
V(k)=\frac{1}{2} s^{2}(k)+\frac{1}{2 \alpha} \Phi_{f}^{T}(k-1) \Phi_{f}(k-1)
$$

Then, $\Delta V(k)$ can be obtained as

$$
\begin{aligned}
\Delta V(k+1) & =V(k+1)-V(k) \\
& =\frac{1}{2} s^{2}(k+1)-\frac{1}{2} s^{2}(k)+\frac{1}{2 \alpha} \Phi_{f}^{T}(k) \Phi_{f}(k) \\
& -\frac{1}{2 \alpha} \Phi_{f}^{T}(k-1) \Phi_{f}(k-1)
\end{aligned}
$$

Let

$$
\Delta \tilde{\theta}_{k}=\frac{1}{2 \alpha} \Phi_{f}^{T}(k) \Phi_{f}(k)-\frac{1}{2 \alpha} \Phi_{f}^{T}(k-1) \Phi_{f}(k-1)
$$

then

$$
\begin{aligned}
& \Delta V(k+1)=\frac{1}{2} s^{2}(k+1)-\frac{1}{2} s^{2}(k)+\Delta \tilde{\theta}_{k} \\
= & \frac{1}{2}(\Delta s(k+1)+s(k))^{2}-\frac{1}{2} s^{2}(k)+\Delta \tilde{\theta}_{k} \\
= & \frac{1}{2} \Delta s(k+1)^{2}+s(k) \Delta s(k+1)+\Delta \tilde{\theta}_{k} \\
= & \frac{1}{2} \Delta s(k+1)^{2}+\Delta \tilde{\theta}_{k} \\
+ & s(k)\left[\Phi_{f}^{T}(k) \xi_{f}(x(k))+w_{f}(k)-Q s(k)-K \operatorname{sign}(s(k))\right] \\
= & \frac{1}{2} \Delta s(k+1)^{2}+s(k) \Phi_{f}^{T}(k) \xi_{f}(x(k))+s(k) w_{f}(k) \\
- & Q s^{2}(k)-K s(k) \operatorname{sign}(s(k))+\Delta \tilde{\theta}_{k}
\end{aligned}
$$

Moroever, $\Delta \tilde{\theta}_{k}$ can be transformed as

$$
\begin{aligned}
& \Delta \tilde{\theta}_{k}=\frac{1}{2 \alpha} \Phi_{f}^{T}(k) \Phi_{f}(k)-\frac{1}{2 \alpha} \Phi_{f}^{T}(k-1) \Phi_{f}(k-1) \\
= & \frac{1}{2 \alpha} \Phi_{f}^{T}(k) \Phi_{f}(k) \\
- & \frac{1}{2 \alpha}\left(\Phi_{f}(k)-\Delta \Phi_{f}(k)\right)^{T}\left(\Phi_{f}(k)-\Delta \Phi_{f}(k)\right) \\
= & \frac{1}{\alpha} \Phi_{f}^{T}(k) \Delta \Phi_{f}(k)-\frac{1}{2 \alpha} \Delta \Phi_{f}^{T}(k) \Delta \Phi_{f}(k)
\end{aligned}
$$

Substituting 25 into 24], we obtain

$$
\begin{aligned}
& \Delta V(k+1)=\frac{1}{2} \Delta s(k+1)^{2}+s(k) \Phi_{f}^{T}(k) \xi_{f}(x(k)) \\
+ & s(k) w_{F}(k)-Q s^{2}(k)-K s(k) \operatorname{sign}(s(k)) \\
+ & \frac{1}{\alpha} \Phi_{f}^{T}(k) \Delta \Phi_{f}(k)-\frac{1}{2 \alpha} \Delta \Phi_{f}^{T}(k) \Delta \Phi_{f}(k) \\
= & \frac{1}{2} \Delta s(k+1)^{2}+s(k) w_{f}(k)-Q s^{2}(k)-K s(k) \operatorname{sign}(s(k)) \\
+ & \Phi_{f}^{T}(k)\left(s(k) \xi_{f}(x(k))+\frac{1}{\alpha} \Delta \Phi_{f}(k)\right) \\
- & \frac{1}{2 \alpha} \Delta \Phi_{f}^{T}(k) \Delta \Phi_{f}(k)
\end{aligned}
$$

By applying the adaptive law 20, equation 26 can be rewritten as

$$
\begin{aligned}
& \Delta V(k+1)=\frac{1}{2} \Delta s(k+1)^{2}+s(k) w_{F}(k)-Q s^{2}(k) \\
& -K s(k) \operatorname{sign}(s(k))-\frac{1}{2 \alpha} \Delta \Phi_{f}^{T}(k) \Delta \Phi_{f}(k)
\end{aligned}
$$

According to 18, we have

$$
\begin{aligned}
|\Delta s(k+1)| & \leq\left|\Phi_{f}^{T}(k) \xi_{f}(x(k))\right|+\left|w_{f}(k)\right| \\
& +|Q s(k)|+|K \operatorname{sign}(s(k))|
\end{aligned}
$$

Furthermore, it's obvious that $\xi_{f}(x(k))$ remains bounded such that

$$
\left\|\xi_{f}(x(k))\right\| \leq \psi_{\xi} \quad \forall k \geq 0
$$

where $\psi_{\xi}$ is a positive constant.

According to (20) and (29) and from $k>N_{0}$, we have

$$
\left|\Delta \theta_{f}(k)\right| \leq \psi_{\theta}|s(k)|
$$

where $\psi_{\theta}$ is positive constant.

Consequently, we can deduce that

$$
\left|\Phi_{f}(k)\right| \leq \psi_{\Phi}|s(k)|
$$


It is obvious that the term $\left|w_{f}(k)\right| \leq \psi_{w}$ where $\psi_{w}$ is a positive constant.

If we define $u_{s g n}=-K \operatorname{sign}(s(k))$ then

$$
\begin{aligned}
|\Delta s(k+1)| & \leq \psi_{\xi} \psi_{\Phi}|s(k)|+\psi_{w}+Q|s(k)|+\left|u_{s g n}\right| \\
& \leq\left(\psi_{\xi} \psi_{\Phi}+Q\right)|s(k)|+\psi_{w}+\left|u_{s g n}\right| \\
& \leq \beta|s(k)|+\left(\psi_{w}+K\right)
\end{aligned}
$$

where $\beta=\left(\psi_{\xi} \psi_{\Phi}+Q\right)$. By taking square from both sides of (32), we can get

$$
\begin{aligned}
|\Delta s(k+1)|^{2} & \leq \beta^{2}|s(k)|^{2}+\left(\psi_{w}+K\right)^{2} \\
& +2 \beta|s(k)|\left(\psi_{w}+K\right)
\end{aligned}
$$

Then,

$$
\begin{aligned}
\frac{1}{2}|\Delta s(k+1)|^{2} & \leq \frac{1}{2} \beta^{2}|s(k)|^{2}+\frac{1}{2}\left(\psi_{w}+K\right)^{2} \\
& +\beta|s(k)|\left(\psi_{w}+K\right)
\end{aligned}
$$

Thus, we can obtain

$$
\begin{aligned}
& \frac{1}{2} \Delta s(k+1)^{2}-Q s^{2}(k)+s(k) u_{s g n} \\
\leq & \frac{1}{2}\left(\psi_{w}+K\right)^{2}+\frac{\beta^{2}}{2}|s(k)|^{2}+\beta|s(k)|\left(\psi_{w}+K\right) \\
- & Q|s(k)|^{2}+K|s(k)|+\psi_{w}|s(k)|-\psi_{w}|s(k)| \\
\leq & \frac{1}{2}\left(\psi_{w}+K\right)^{2}+(\beta+1)|s(k)|\left(\psi_{w}+K\right)-\psi_{w}|s(k)| \\
+ & \left(\frac{1}{2} \beta^{2}-Q\right)|s(k)|^{2}
\end{aligned}
$$

If we choose

$$
Q>\frac{1}{2} \beta^{2}
$$

and

$$
\begin{aligned}
K & =\left(-(\beta+1)+\sqrt{\left[(\beta+1)^{2}+2\left(Q-\frac{1}{2} \beta^{2}\right)\right]}\right)|s(k)| \\
& -\psi_{w}
\end{aligned}
$$

then

$$
\begin{aligned}
\frac{1}{2}\left(\psi_{w}+K\right)^{2} & +(\beta+1)|s(k)|\left(\psi_{w}+K\right) \\
& +\left(\frac{1}{2} \beta^{2}-Q\right)|s(k)|^{2}=0
\end{aligned}
$$

Therefore, 27 can be expressed as

$$
\begin{aligned}
\Delta V(k+1) & \leq s(k) w_{f}(k)-\psi_{w}|s(k)| \\
& -\frac{1}{2 \alpha} \Delta \Phi_{f}^{T}(k) \Delta \Phi_{f}(k)
\end{aligned}
$$

It's clear that $s(k) w_{f}(k)-\psi_{w}|s(k)| \leq 0$ since $\left|w_{f}(k)\right| \leq \psi_{w}$, furthermore $\Delta \Phi_{f}^{T}(k) \Delta \Phi_{f}(k) \geq 0$, then

$$
\Delta V(k+1) \leq 0
$$

By using the Barbalat's lemma [23], we can readily prove that $s(k) \rightarrow 0$ as $k \rightarrow+\infty$ thus $\lim _{k \rightarrow+\infty} e(k)=0$.

\section{Simulation results}

To illustrate the above design approach, a 3D Henon chaotic model is considered.

The model for the discrete-time 3D Henon map is given as [22]

$$
\left\{\begin{aligned}
x_{1}(k+1) & =x_{2}(k) \\
x_{2}(k+1) & =x_{3}(k) \\
x_{3}(k+1) & =-0.2 x_{1}(k)-0.3 x_{2}(k)-1.65 x_{3}(k)-x_{3}^{2}(k) \\
& +u(k)
\end{aligned}\right.
$$

The non controlled $(u(k)=0)$ Henon map has a chaotic strange attractor. The result of computation is shown in figure 1 where $\left[x_{1}(0), x_{2}(0), x_{3}(0)\right]^{T}=$ $[0.1,0,0.1]^{T}$.

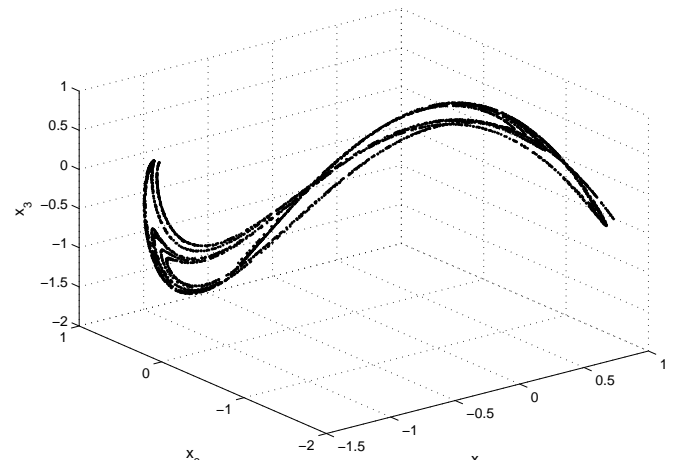

Figure 1: Hénon map attractor.

In order to control this autonomous chaotic system, controller $u(k)$, given by $(8)$, is applied at $k=60$ to reach the desired trajectory $x_{d}(k)=[0,0,0]^{T}$.

For the estimation of $f(x(k))$, we consider five fuzzy levels, i.e. NB, NS, EZ, PS and PB on $x_{1}, x_{2}$ and $x_{3}$. We use fuzzy logic systems with center-average defuzzifier, product inference, singleton fuzzifier and Gaussian membership functions to approximate $f(x(k))$. Slopes $c_{i}$ are chosen as $c_{1}=0.1$ and $c_{2}=0.9$.

In figure 2, we represent the evolution of system states which converge rapidly to the desired values. Figure 3 represent the sliding surface and the control signal whose amplitude is always within an acceptable range compared to the system states.
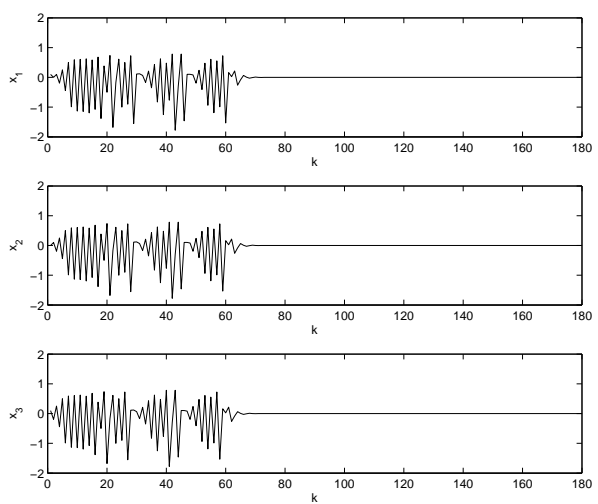

Figure 2: Evolution of system states 

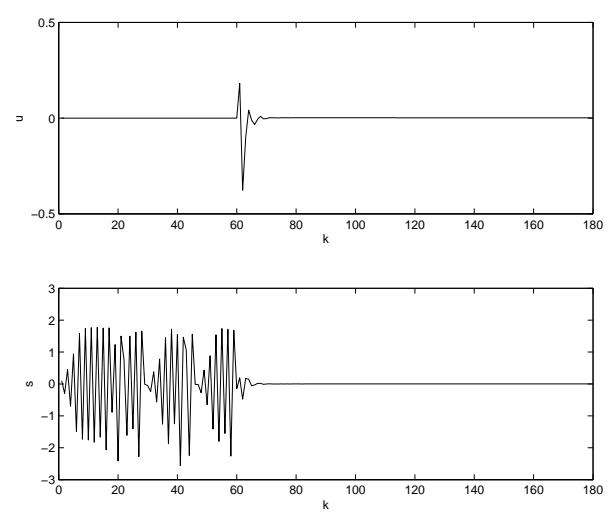

Figure 3: Representation of the sliding surface and the fuzzy sliding mode controller.

In order to illustrate the robustness of our design, a white gaussian noise with variance equal to 0.01 has been considered as an external disturbance. The control objective has been achieved as is illustrated in figures 4 and 5. Thus, the robustness of the discrete fuzzy sliding mode controller is proved.
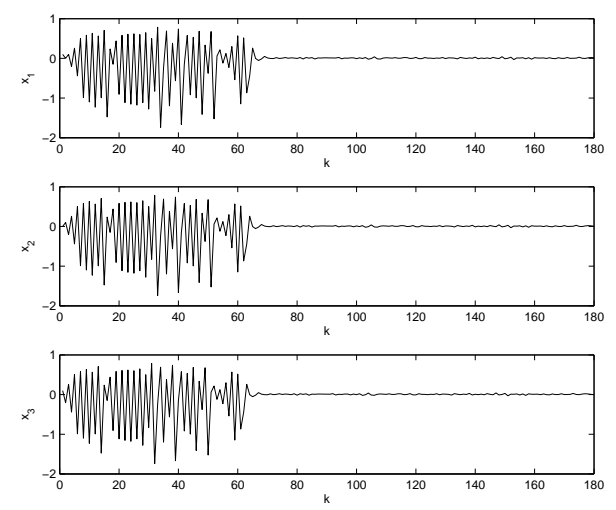

Figure 4: Evolution of system states in presence of a white gaussian noise.
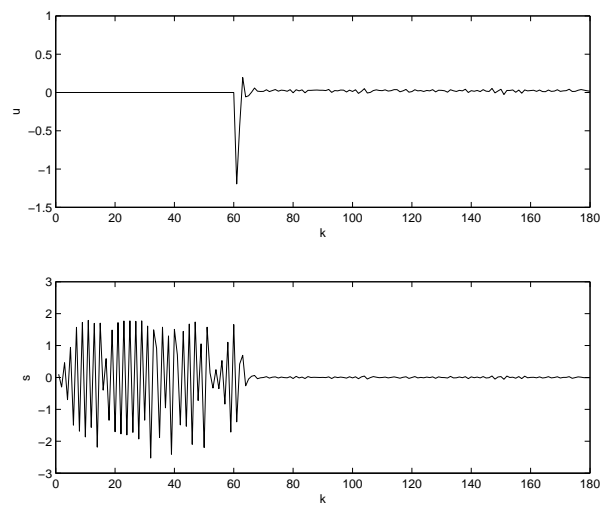

Figure 5: Representation of the sliding surface and the fuzzy sliding mode controller.

\section{Conclusion}

In this paper, an adaptive discrete-time fuzzy sliding mode controller is proposed. This controller, inherits the advantages of both sliding mode control and fuzzy systems. It has been proposed for stable control of a class of chaotic systems. The sliding mode control is proposed as a robust method to control nonlinear and uncertain systems. Consequence parameters of fuzzy control rules have been adjusted on-line in order to guarantee the reaching condition. Simulation results of a 3D Henon chaotic model show the applicability and the effectiveness of the proposed approach.

Conflict of Interest The authors declare no conflict of interest.

\section{References}

1. E. Ott, C. Grebogi, J. A. Yorke, "Controlling chaos”, Physical Review Letters, 64, 1196-1199, 1990.

2. X. Tan , J. Zhang, Y. Yang, "Synchronizing chaotic systems using backstepping design", Chaos, Solitons and Fractals, 16, 37-45, 2003.

3. J. H. Park, "Synchronization of Genesio chaotic system via backstepping approach", Chaos, Solitons and Fractals, 27(5), 1369-1375, 2006.

4. H. Adelipoor, O.A. Babaei, "Stability and Tracking in the New Chaotic System Using Backstepping Method", J. Basic. Appl. Sci. Res., 3, 446452, 2013.

5. H.Zhang, H. Quin, G. Chen, "Adaptive control of chaotic systems with uncertainties", Int. J. Bifurcation and Chaos, 8(10), 2041-2046, 1998.

6. M. Feki, "Model-independent adaptive control of chua's system with cubic nonlinearity", Int. J. Bifurcation and Chaos, 14(12), 4249-4263, 2004.

7. M. P. Aghababa, B. Hashtarkhani, "Synchronization of Unknown Uncertain Chaotic Systems Via Adaptive Control Method", Journal of Computational and Nonlinear Dynamics, 10(5), 051004, 2015.

8. H.T. Yau, C.K. Chen, C. Li Chen, "Sliding mode control of chaotic systems with uncertainties", Int. J. Bifurcation and Chaos, 10(5), 1139-1147, 2000.

9. J. J. Yan, Y. S. Yang, T. Y. Chiang, C. Y. Chen, "Robust synchronization of unified chaotic systems via sliding mode control", Chaos, Solitons and Fractals, 34(3), 947-954, 2007.

10. M. Feki, "Sliding mode control and synchronization of chaotic systems with parametric uncertainties", Chaos Solitons and Fractals, 41(3), 1390-1400, 2009. 
11. K. Furuta, "Sliding mode control of a discrete systems", Systems and Control Letters, 14(2), 145-152, 1990.

12. W. Gao, Y. Wang, A. Homaifa, "Discrete-time variable structure control systems", IEEE Trans. Ind. Electronics, 42(2), 117-122, 1995.

13. H. Sira-Ramirez, "Non-linear discrete variable structure systems in quasi-sliding mode", International Journal of Control, 54(5), 1171-1187, 1991.

14. S.Z. Sarpturk, Y. Istefanopulos, O. Kaynak, “On the stability of discrete-time sliding mode systems", IEEE Transaction Automatic Control, AC-32(10), 930-932, 1987.

15. J.Y. Chen, "Rule regulation of fuzzy sliding mode controller design: direct adaptive approach", Fuzzy sets and systems, 120, 159-168, 2001.

16. O. Kaynak, "Fuzzy adaptive sliding mode control of a direct drive robot, Robotics and Autonomous Systems”, 19, 215-227, 1996.

17. H. Medhaffar, T. Damak, N. Derbel, "A decoupled fuzzy indirect adaptive sliding mode con- troller with application to robot manipulators", IJMIC, 1(1), 23-29, 2006.

18. H. Medhaffar, T. Damak, N. Derbel, "Direct adaptive fuzzy moving sliding mode controller design for robotic manipulators", IJCIA, 5(1), 120, 2005.

19. L.A. Zadeh, "Outline of a new approach to the analysis of complex systems and decision processes", IEEE Trans. Systems, Man. cybernetics, 3(1), 28-44, 1973.

20. L.X. Wang, Adaptive fuzzy systems and control, Englewood Cliffs, NJ: Prentice-Hall, 1994.

21. B. Yoo, W. Ham, "Adaptive fuzzy sliding mode control of nonlinear systems", IEEE Trans. Fuzzy systems, 6(2), 315-321, 1998.

22. S. V. Gonchenko, I. I. Ovsyannikov, C. Simo, D. Turaev, "Three Dimensional Hénon-like Maps and Wild Lorenz-like Attractors", International Journal of Bifurcation and Chaos, 15 (11), 34933508, 2005.

23. J. J. E. Slotine, W. Li, Applied Nonlinear Control, Englwood Cliffs, NJ:Prentice Hall, 1991. 